This item was submitted to Loughborough's Research Repository by the author.

Items in Figshare are protected by copyright, with all rights reserved, unless otherwise indicated.

\title{
Fast optimization of cache-enabled Cloud-RAN using determinantal point process
}

PLEASE CITE THE PUBLISHED VERSION

https://doi.org/10.1016/j.phycom.2021.101292

PUBLISHER

Elsevier

VERSION

AM (Accepted Manuscript)

\section{PUBLISHER STATEMENT}

This paper was accepted for publication in the journal Physical Communication and the definitive published version is available at https://doi.org/10.1016/j.phycom.2021.101292.

LICENCE

CC BY-NC-ND 4.0

\section{REPOSITORY RECORD}

Bsebsu, Ashraf, Gan Zheng, and Sangarapillai Lambotharan. 2021. "Fast Optimization of Cache-enabled Cloud-ran Using Determinantal Point Process". Loughborough University. 


\title{
Fast Optimization of Cache-Enabled Cloud-RAN Using Determinantal Point Process
}

\author{
Ashraf Bsebsu, Gan Zheng and Sangarapillai Lambotharan \\ Signal Processing and Networks Research Group, Loughborough University, Loughborough, \\ LE11 3TU, UK
}

\begin{abstract}
Cloud radio access network (Cloud-RAN) has been considered as a potential candidate for the next generation of radio access networks. It addresses many challenges in terms of flexibility, scalability, radio resource management and energy efficiency. Caching popular contents at radio remote heads (RRHs) plays an important role for reducing fronthaul traffic congestion and delay in cache-enabled Cloud-RAN. Although, mathematical optimization methods have shown to be providing numerical solutions for addressing key signal processing issues in Cloud-RAN, the exponential complexity hinders their application in practice, particularly in large networks. Learning-based methods have become attractive to overcome the complexity issues associated with the mathematical optimization methods. Several subset selection problems have been formulated as a mixed-integer non linear program (MINLP) in wireless networks. Determinantal point process (DPP) is a probabilistic model of choosing two similar items which are negatively correlated. In this paper, we propose a DPP basedlearning (DPPL) framework to obtain a subset of admitted users for cacheenabled Cloud-RAN with limited fronthaul capacity. The formulated problem of minimizing the total network cost including power and fronthaul cost while admitting as many users as possible is converted into mixed-integer second order cone programming (MI-SOCP). The subset of admitted users is obtained by learning the quality-diversity trade-off of the DPP using the optimal subsets of admitted users which are obtained by the optimization approach. We then propose an optimization algorithm to determine the beamforming and the base station-user allocation for the obtained subset of admitted users. We provide numerical results to assess the performance and complexity of the proposed DPPL algorithm and compare it with its optimization counterpart. The results reveal that the proposed DPPL can achieve a comparable performance with much lower complexity.
\end{abstract}

Keywords: Cloud-RAN, downlink beamforming, machine learning, determinantal point process, caching, mixed-integer second order cone programming.

Preprint submitted to Elsevier

December 25, 2020 


\section{Introduction}

Cloud radio access network (Cloud-RAN) is emerging as a new architecture in the fifth generation $(5 \mathrm{G})$ network. It brings new advantages over traditional radio access network by providing and supporting multi-cell collaborative signal 5 processing as well as enhancing network resource sharing. The radio and baseband processing functions in the Cloud-RAN are carried out in two separated units namely remote radio head $(\mathrm{RRH})$ and base band unit (BBU) respectively. RRHs are connected to a centralized BBU through fronthaul links 11. As signals are processed by a cloud processor, this will enable cooperative transmission and radio resource allocation management to be implemented. Since the data volume of a user is increasing due to various quality of service (QoS) requirements, the fronthaul links are getting congested. Cache-enabled Cloud-RANs can significantly alleviate the traffic congestion and enhance the communication efficiency and delay performance 2 by caching popular requested content

15 files at RRHs. Caching and cooperation transmission in Cloud-RAN have been considered as an effective way to tackle the challenge of fronthaul capacity limitation.

In the literature, Cloud-RANs have been investigated from various aspects.

20 For example, the works [3], 4, [5], 6] and [7] studied the energy consumption minimization aspect. The majority of these works have implemented traditional optimization approaches for designing algorithmic solutions. These approaches have played an important role for delivering an effective numerical solutions and theoretical analysis to many problems, however, implementing such solutions are

25 not practical in real systems due to the fact that some of these algorithms are time costly because of the nature of its computational demands [8]. Machine learning has been introduced as an alternative tool to replace traditional optimization tool for obtaining solutions. Since the fundamentals of the wireless networks are based on conventional probabilistic models, for example, channel, 30 noise and interference, machine learning is converting this model-driven approach to data-driven simulation based approach by using large sets of available data from real networks 9. A wide range of resource management problems in wireless network such as user association and base station selection appear as mixed-integer nonlinear programming (MINLP). These problems are also known

35 as subset selection problems where a subset that optimizes a certain objective function needs to be selected from an underlying set. Complicated optimization algorithms are commonly used such as branch and bound algorithm (BnB) for obtaining the global optimal solutions however with exponential complexity.

Subset selection problems in machine learning are presented in different contexts. The main concept was to balance the quality and the diversity of the items in the subset, i.e., to choose good quality items from the underlying set that are also non-overlapping in terms of their features. Modeling the diversity between the items in a subset selection problem leads us to exploit the advantages that

45 some probabilistic models can provide. Determinantal point processes (DPPs) 
are a natural choice for inducing diversity or negative correlation between the items in a subset. In machine learning applications, DPPs in finite space have been discussed as an appropriate probabilistic model that provide a trade-off between the quality and diversity to solve subset selection problems [10.

In wireless cellular networks, majority of the works of DPPs usage have focused on stochastic geometry based modeling and analysis. For example, the work in 11] proposed DPPs to demonstrate the accuracy of modeling the base station locations based on spatial repulsion over traditional model using popular

${ }_{55}$ Poisson point process. The authors in 12 proposed special type of DPPs called Ginibre point process to model the deployment of the wireless base stations where a computable representation for the coverage probability was derived. The finite DPPs and associated data driven learning framework were proposed in 13, where the trained DPPs have been used to imitate some properties for 60 some hard-core point processes used in wireless networks. In [14, the authors developed DPP based-learning framework to solve subset selection problem in wireless networks where two different mathematical tools (stochastic geometry and machine learning) are jointly applied.

In this paper, we propose a DPP based-learning (DPPL) framework to obtain a subset of admitted users for cache-enabled Cloud-RAN with limited fronthaul capacity. The objective is to minimize the total network cost under a certain constraints while admitting as many users as possible. A solution of integer values 1 and -1 will be assigned to the users. The users with high values will be 70 deemed as admitted users whereas the users with low values will be deemed as dropped users. The proposed DPPL framework will select the subset of simultaneously admitted users by balancing between the quality and diversity, i.e., selecting the users that have a good quality in terms of signal to interference plus noise ratio with some degree of channel un-correlation between those users 75 to avoid the interference. We treated the subset of admitted users as a DPP over a ground set of the users in a given network. We trained the DPPs with a sequence of optimal subsets of admitted users obtained by using the optimization approach. In addition, we developed an optimization algorithm to obtain the beamforming and base station-user allocation given the selected subset of users.

This paper is organized as follows. The network model and problem formulation are presented in Section II. In Section III, a conventional branch and bound algorithm is described. A brief introduction to determinantal processes 85 is presented in Section IV. A DPPL framework is proposed in Section V. Simulation results are provided in Section VI, followed by conclusions in Section VII.

Notations: We denote matrices and vectors with boldface upper-case and boldface lower-case respectively. We denote the sets of complex and real num90 bers as $\mathbb{C}$ and $\mathbb{R}$ respectively. $E[$.$] denotes the expected value of a random$ variable. $\mathcal{C N}\left(\mu, \sigma^{2}\right)$ represents the complex Gaussian distribution with mean $\mu$ 
and variance $\sigma^{2}$. The conjugate transpose of a vector is denoted as $(.)^{H}$. $\mathbf{0}_{l}$ and $\mathbf{1}_{l}$ represent the $l$-length all zeros and $l$-length all ones vectors respectively. $\operatorname{Re}\{$.$\} and \operatorname{Im}\{$.$\} represent the real and imaginary parts of a complex variable,$ respectively.

\section{NETWORK MODEL AND PROBLEM FORMULATION}

\subsection{System Model}

We consider a cache-enabled Cloud-RAN in downlink transmission with $K$ users and $L$ RRHs. All RRHs are connected to the BBU through limited capacity fronthaul links. We consider a single antenna at each user terminal and denote the set of users $\mathcal{K}=\{1,2, \cdots, K\}$ as shown in Figure 1. In addition, we assume that each RRH is equipped with $N$ antennas and has finite capacity for local storage. We denote the set of RRHs as $\mathcal{L}=\{1,2, \cdots, L\}$. Furthermore, we assume that a library of $F$ contents can be accessed through the BBU to

serve user's request. Each user can be served by a single RRH or a cluster of RRHs in a cooperative manner.

We define the beamforming vector to transmit the data from $\mathrm{RRH} l$ to user $k$ as $\mathbf{w}_{l, k} \in \mathbb{C}^{N \times 1}$. The received signal at the user $k$ can be expressed as

$$
y_{k}=\sum_{l=1}^{L} \mathbf{h}_{l, k}^{H} \mathbf{w}_{l, k} s_{k}+\sum_{i=1, i \neq k}^{K} \sum_{l=1}^{L} \mathbf{h}_{l, k}^{H} \mathbf{w}_{l, i} s_{i}+n_{k}, \forall k \in \mathcal{K}
$$

where $\mathbf{h}_{l, k} \in \mathbb{C}^{N \times 1}$ is the channel vector from $\mathrm{RRH} l$ to user $k, s_{k} \in \mathbb{C}$ denotes a unit power transmit data symbol to the user $K$, i.e., $E\left[\left|s_{k}\right|^{2}\right]=1, n_{k} \in \mathbb{C}$ is additive Gaussian noise with distribution of $\mathcal{C N}\left(0, \sigma_{k}^{2}\right), k \in \mathcal{K}$.

The signal to interference plus noise ratio (SINR) at the receiver end of the user $k$ can be expressed as

$$
S I N R_{k}=\frac{\left|\sum_{l=1}^{L} \mathbf{h}_{l, k}^{H} \mathbf{w}_{l, k}\right|^{2}}{\sum_{i=1, i \neq k}^{K}\left|\sum_{l=1}^{L} \mathbf{h}_{l, k}^{H} \mathbf{w}_{l, i}\right|^{2}+\sigma_{k}^{2}}, \forall k \in \mathcal{K} .
$$

We define $\mathbf{B} \in\{0,1\}^{L \times K}$ as the base station-user association matrix, where $b_{l, k}=1$ indicates that the RRH $l$ serves the user $k$ and $b_{l, k}=0$ otherwise. The relation between $b_{l, k}$ and $\mathbf{w}_{l, k}$ is described as:

$$
\left\{\begin{array}{l}
b_{l, k}=0 \Leftrightarrow \mathbf{w}_{l, k}=\mathbf{0}, \forall l \in \mathcal{L}, \forall k \in \mathcal{K}, \\
b_{l, k}=1 \Leftrightarrow \mathbf{w}_{l, k} \neq \mathbf{0}, \forall l \in \mathcal{L}, \forall k \in \mathcal{K} .
\end{array}\right.
$$

\subsection{Cache Model and Request Model}

We consider a library of $F$ contents with identical normalized size and denote the set of contents as $\mathcal{F}=\{1,2, \cdots, F\}$. We define the caching placement matrix $\mathbf{C} \in\{0,1\}^{F \times L}$, where $c_{f, l}=1$ denote that the the $f^{t h}$ content is cached 


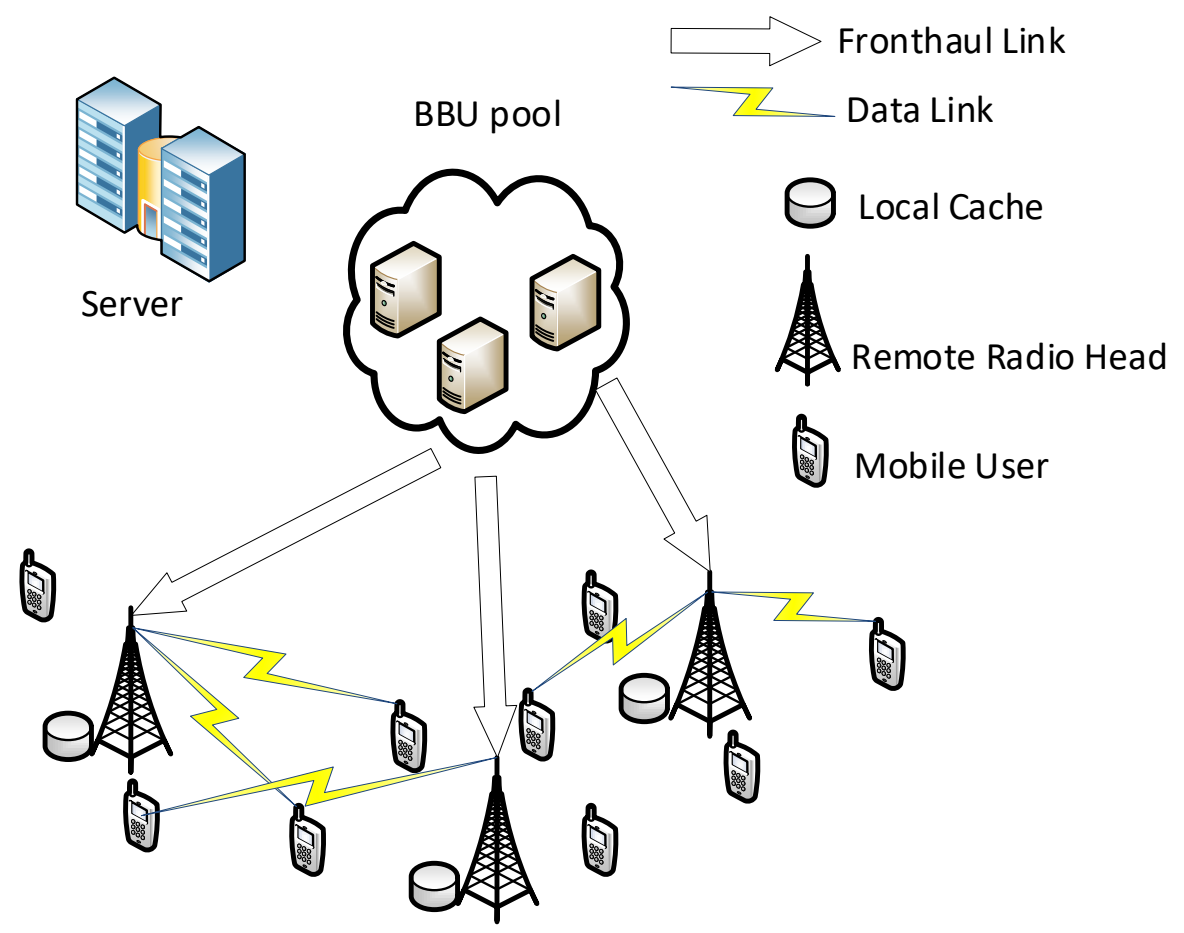

Figure 1: System model of Cache-enabled downlink Cloud-RAN with limited fronthaul capacity. 

each RRH as $Y_{l}$ and $Y_{l}<<F$. The total number of contents that can be stored in the local storage is limited by its maximum storage capacity $\sum_{f=1}^{F} c_{f, l} \leq Y_{l}$. Due to a time scale variation between short-term transmission and long-term caching placement, we assume that caching placement matrix is known a prior 125 and fixed. Let us consider that each user requests a content file and define user requests matrix as $\mathbf{R} \in \mathbb{R}^{F \times K}$, where $r_{f, k}=B_{k} \log _{2}\left(1+\gamma_{k}\right)$ means that the $k^{t h}$ user requests the $f^{t h}$ content with a target SINR $\gamma_{k}$ and $r_{f, k}=0$ otherwise, where $B_{k}$ is the user bandwidth. If the requested content of the user $k$ is cached in the RRH $l$, the user can get the content directly from RRH $l$ without using BBU through the fronthaul link.

\subsection{Network Cost Model}

We consider the total network cost as the sum of the costs of transmission power and fronthaul. We model the transmission power cost using the beamforming vectors of RRHs as

$$
C_{p}=\sum_{l=1}^{L} \sum_{k=1}^{K}\left\|\mathbf{w}_{l, k}\right\|_{2}^{2} .
$$

The fronthaul cost is modeled as [15]:

$$
C_{B}=\sum_{l=1}^{L} \sum_{k=1}^{K} \sum_{f=1}^{F} b_{l, k}\left(1-c_{f, l}\right) r_{f, k} .
$$

The total network cost can be expressed as:

$$
C_{N}=C_{p}+\eta C_{B}
$$

135

where $\eta$ is a weighting factor $(\eta>0)$.

To enable the joint processing, we consider that the channel state information, user request matrix and caching placement matrix are available at the BBU.

\subsection{Problem Formulation}

We formulate the problem as minimizing the total network cost for the cacheenabled Cloud-RAN while admitting as many users as possible and satisfying the constraints of transmission power, admitted users' quality of service (QoS) and the fronthaul capacity. By adopting the approach in [16, we can reformulate the two-stage problem into one-stage problem of joint beamforming and admission control (JBAC) by introducing two small positive parameters $\alpha$ and $\beta$, which are essential for the penalty for user admission and feasibility guarantee, respectively. The JBAC problem is expressed as: 


$$
\begin{aligned}
& \min _{\left\{\mathbf{w}_{l, k}^{*}, b_{l, k}^{*}, a_{k}^{*}\right\}} \quad \alpha C_{N}+(1-\alpha) \sum_{k=1}^{K}\left(a_{k}-1\right)^{2} \\
& \text { s.t. } \quad \sum_{k=1}^{K}\left\|\mathbf{w}_{l, k}\right\|_{2}^{2} \leq P_{l}^{M}, \forall l \in \mathcal{L}, \\
& \sum_{k=1}^{K} \sum_{f=1}^{F} b_{l, k}\left(1-c_{f, l}\right) r_{f, k} \leq R_{l}^{M}, \\
& \left\|\mathbf{w}_{l, k}\right\|_{2}^{2} \leq b_{l, k} P_{l}^{M}, \forall l \in \mathcal{L}, \forall k \in \mathcal{K}, \\
& b_{l, k} \leq \frac{a_{k}+1}{2}, \forall l \in \mathcal{L}, \forall k \in \mathcal{K}, \\
& \sum_{l=1}^{L} b_{l, k} \geq \frac{a_{k}+1}{2}, \forall l \in \mathcal{L}, \forall k \in \mathcal{K}, \\
& b_{l, k} \in\{0,1\}, a_{k} \in\{-1,1\}, \forall l \in \mathcal{L}, \forall k \in \mathcal{K}, \\
& \quad \frac{\left|\sum_{l=1}^{L} \mathbf{h}_{l, k}^{H} \mathbf{w}_{l, k}\right|^{2}-\beta^{-1}\left(a_{k}-1\right)}{\sum_{i=1, i \neq k}^{K}\left|\sum_{l=1}^{L} \mathbf{h}_{l, k}^{H} \mathbf{w}_{l, i}\right|^{2}+\sigma_{k}^{2}} \geq \gamma_{k}, \forall k \in \mathcal{K},
\end{aligned}
$$

where $P_{l}^{M}$ represents the power budget of $\mathrm{RRH} l, R_{l}^{M}$ represents the maximum 150 fronthaul capacity link of the RRH $l, \gamma_{k}$ is the target SINR of user $k, a_{k}$ is the user access indicator, where $a_{k}=1$ indicates that the user $k$ accesses the network and deemed as admitted and $a_{k}=-1$ otherwise. The QoS constraint (7h) ensures that user $k$ achieves the target SINR $\gamma_{k}$. The constraint $(7 \mathrm{~b})$ means that the total transmit power of RRH $l$ is below the maximum transmit power.

155 The fronthaul constraint $\sqrt{7 \mathrm{c}})$ indicates that the data transfer rate from BBU to RRH $l$ is below the maximum fronthaul capacity. The constraint $(7 \mathrm{~d})$ is to ensure that when $b_{l, k}=0, \mathbf{w}_{l, k}=0$. The constraint $(7 \mathrm{~g})$ indicates that the value of $b_{l, k}$ can only be 0 or 1 and the value of $a_{k}$ is -1 or 1 . The constraints (7e) and (7f) ensure that when user is dropped, i.e., $a_{k}=-1, \forall k \in \mathcal{K}$, both $b_{l, k}=0$ and $\mathbf{w}_{l, k}=\mathbf{0}, \forall l \in \mathcal{L}$.

The problem (7) is a non-convex problem due to the discrete values of constraint $(7 \mathrm{~g})$ and the QoS constraint $(7 \mathrm{~h})$. Since the rotation of each element of $\mathbf{w}_{l, k}$ by an arbitrary phase angle, i.e., $\mathbf{w}_{l, k} \exp (j \phi)$ for any $\phi$, will have no impact on the network power consumption and the QoS constraints, i.e., (4) and (2), we manipulate the QoS constraint $(7 \mathrm{~h})$ to a more tractable second order cone programming (SOCP) form. We reformulated the QoS constraint $7 \mathrm{~h}$ into a standard SOCP constraint as 


$$
\begin{aligned}
& \operatorname{Re}\left\{\sum_{l=1}^{L} \mathbf{h}_{l, k}^{H} \mathbf{w}_{l, k}-\beta^{-1}\left(a_{k}-1\right)\right\} \geq \\
& \sqrt{\gamma_{k}\left\{\sum_{i=1, i \neq k}^{K}\left|\sum_{l=1}^{L} \mathbf{h}_{l, k}^{H} \mathbf{w}_{l, i}\right|^{2}+\sigma_{k}^{2}\right\}}, \\
& \operatorname{Im}\left\{\sum_{l=1}^{L} \mathbf{h}_{l, k}^{H} \mathbf{w}_{l, k}-\beta^{-1}\left(a_{k}-1\right)\right\}=0 .
\end{aligned}
$$

The value of $\beta$ can be determined as $0<\beta \leq \min _{k \in K} \frac{2}{\sqrt{\gamma_{k}\left(\sum_{l=1}^{L} P_{l}^{M} \sum_{l=1}^{L}\left\|h_{l, k}\right\|^{2}+\sigma_{k}^{2}\right)}}$, which satisfies the QoS constraint explicitly when $a_{k}=-1$.

We can rewrite the problem (7) with reformulated QoS constraints as follow

$$
\begin{aligned}
& \min _{\left\{\mathbf{w}_{l, k}^{*}, b_{l, k}^{*}, a_{k}^{*}\right\}} \alpha C_{N}+(1-\alpha) \sum_{k=1}^{K}\left(a_{k}-1\right)^{2} \\
& \text { s.t. } \quad \sum_{k=1}^{K}\left\|\mathbf{w}_{l, k}\right\|_{2}^{2} \leq P_{l}^{M}, \forall l \in \mathcal{L}, \\
& \sum_{k=1}^{K} \sum_{f=1}^{F} b_{l, k}\left(1-c_{f, l}\right) r_{f, k} \leq R_{l}^{M}, \\
& \left\|\mathbf{w}_{l, k}\right\|_{2}^{2} \leq b_{l, k} P_{l}^{M}, \forall l \in \mathcal{L}, \forall k \in \mathcal{K}, \\
& b_{l, k} \leq \frac{a_{k}+1}{2}, \forall l \in \mathcal{L}, \forall k \in \mathcal{K}, \\
& \sum_{l=1}^{L} b_{l, k} \geq \frac{a_{k}+1}{2}, \forall l \in \mathcal{L}, \forall k \in \mathcal{K}, \\
& b_{l, k} \in\{0,1\}, a_{k} \in\{-1,1\}, \forall l \in \mathcal{L}, \forall k \in \mathcal{K}, \\
& \operatorname{Re}\left\{\sum_{l=1}^{L} \mathbf{h}_{l, k}^{H} \mathbf{w}_{l, k}-\beta^{-1}\left(a_{k}-1\right)\right\} \geq \\
& \quad \sqrt{\gamma_{k}\left\{\sum_{i=1, i \neq k}^{K}\left|\sum_{l=1}^{L} \mathbf{h}_{l, k}^{H} \mathbf{w}_{l, i}\right|^{2}+\sigma_{k}^{2}\right\},} \\
& \operatorname{Im}\left\{\sum_{l=1}^{L} \mathbf{h}_{l, k}^{H} \mathbf{w}_{l, k}-\beta^{-1}\left(a_{k}-1\right)\right\}=0 .
\end{aligned}
$$

Now, our aim is to convert the problem (9) into a mixed-integer programming form (MIP). Hence, we introduce a vector $\mathbf{b}_{k} \in \mathbb{R}^{L \times 1}, k=1, \ldots, K$, which can 
take one of the following combinations [17]:

$$
\mathbf{b}_{k} \in\left\{\left[\begin{array}{l}
0 \\
0 \\
\cdot \\
\dot{0}
\end{array}\right],\left[\begin{array}{l}
1 \\
0 \\
\cdot \\
\cdot \\
0
\end{array}\right],\left[\begin{array}{l}
0 \\
1 \\
\cdot \\
0
\end{array}\right],\left[\begin{array}{l}
1 \\
1 \\
\cdot \\
\cdot \\
0
\end{array}\right], \ldots,\left[\begin{array}{l}
1 \\
1 \\
\cdot \\
\cdot \\
1
\end{array}\right]\right\} .
$$

If the $l^{\text {th }}$ element of $\mathbf{b}_{k}$ is one, the corresponds $k^{\text {th }}$ user is served by the $l^{\text {th }}$ $\mathrm{RRH}$ and $a_{k}=1$. The only case that the $k^{t h}$ user is dropped, i.e., $a_{k}=-1$ is when all the elements of a vector $\mathbf{b}_{k}$ are zeros.

\section{THE CONVENTIONAL BRANCH AND BOUND APPROACH}

The formulation of the problem $(9)$ is a combinatorial optimization problem. An exhaustive search method is usually required to solve the MIP problem and 175 to obtain the optimal solution. The complexity of this method increases exponentially with the number of variables. A conventional BnB algorithm is used to determine the optimal solution for the MIP problem with low complexity in terms of time and storage required for computation compared to exhaustive search method. The BnB method can be described in two main steps: the first feasible set of the problem is divided into small problem subsets according to different combinations. The second step is bounding, where the evaluation of the lower bounds of those subsets is conducted. Tree structure will be created accordingly by dividing those subsets into smaller subsets. Some of the branches will be removed due to infeasibility or their optimal objective values are greater than the best-known global objective value of the minimization problem. The global lower bound will be updated at each level only if the current global lower bound is greater than the minimum of the lower bounds of all subsets at the level. By relaxing the constraints in (9g), the problem in $(9)$ is a mixed-integer second order cone programming (MI-SOCP)

190 and the proposed optimal algorithm based on $\mathrm{BnB}$ method in 18 can be used to obtain the optimal solution for the JBAC problem in (9). The optimal solution obtained includes the subset of admitted users $\left\{a_{k}^{*}\right\}$ which will be used later for training and comparison purposes.

\section{PRELIMINARIES OF DETERMINANTAL PROCESSES}

In this section, we provide a concise introduction to determinantal process on the discrete finite space. Let us consider discrete space $\mathcal{K}$ with finite cardinality $K<\infty$. A point process $\mathcal{P}$ on the space $\mathcal{K}$ is basically defined as the probability measure on $2^{\mathcal{K}}$, the set of all subsets of $\mathcal{K}$. A sample from $\mathcal{P}$ could be an empty set, the entirety of $\mathcal{K}$ or anything in between. Let us denote $\mathbf{Y}$ as a random set drawn according to $\mathcal{P}$, then the detrminantal point process (DPP) is a measure 
of likelihood for selecting subsets of a random set $\mathbf{Y}$. For every $A \subseteq \mathbf{Y}, \mathrm{DPP}$ can be defined as

$$
\mathcal{P}(A \subseteq \mathbf{Y})=\operatorname{det}\left(\kappa_{A}\right),
$$

where $\kappa_{A} \equiv\left\{\kappa_{i, j}\right\}_{i, j \in A}$ denotes the restriction of $\kappa \in \mathbb{R}^{K \times K}$ to the entries indexed by elements of $A$, and $\operatorname{det}\left(\kappa_{\emptyset}\right)=1 . \quad \kappa$ is a marginal kernel matrix which contains all needed information to quantify the probability measure for any subset $A, A \subseteq \mathbf{Y}$. $\kappa$ is also a positive semidifinite matrix in which all its eigenvalues are less than or equal to 1 . For learning real data, it is more useful to represent the DPPs using an alternative formalism known as the $L$-ensemble which was introduced in [19]. In the $L$-ensemble, the DPP is not defined through the marginal kernel $\kappa$ but through a real, symmetric matrix $\mathrm{E}$. Thus, we can rewrite the DPP using $€$ matrix indexed by elements of $\mathcal{K}$ as follow

$$
\mathcal{P}_{\mathrm{E}}(Y) \equiv \mathcal{P}_{\mathrm{E}}(\mathbf{Y}=Y)=\frac{\operatorname{det}\left(\mathrm{Ł}_{Y}\right)}{\operatorname{det}(\mathrm{E}+I)},
$$

where $I$ is a $K \times K$ identity matrix, $\mathcal{P}_{\mathrm{E}}(Y)$ is normalized due to $\sum_{Y \subset \mathcal{K}} \operatorname{det}\left(\mathrm{L}_{Y}\right)=$ $\operatorname{det}(\mathrm{E}+I)$ (see [10, Theorem 2.1] for proof). The $\mathrm{L}_{Y}$ matrix can be expressed by element indexes as $\mathrm{E}_{Y}=\left[\mathrm{E}_{i, j}\right]_{i, j \in Y}$.

Since $\mathrm{E}$ is a real and symmetric matrix, we can compute its eigendecomposition as $\mathrm{L}=\sum_{k=1}^{K} \lambda_{k} \mathbf{v}_{k} \mathbf{v}_{k}^{\top}$, where $\lambda_{k}$ represents the eigenvalues and $\mathbf{v}_{k}$ represents the orthonormal sequence of eigenvectors corresponding to the eigenvalues. Similarly, the kernel $\kappa$ is also a real and symmetric matrix. By preserving the eigenvectors and re-scaling the eigenvalues of $\mathrm{E}$, the eigendecomposition of $\kappa$ is $\kappa=\sum_{k=1}^{K} \frac{\lambda_{k}}{\lambda_{k}+1} \mathbf{v}_{k} \mathbf{v}_{k}^{\top}$. An $L-$ ensemble is a DPP and its marginal kernel (see [10, Theorem 2.2] for proof). The relation between $L$-ensemble matrix $\mathrm{E}$ and the kernel matrix $\kappa$ as follows

$$
\kappa=\mathrm{E}(\mathrm{E}+I)^{-1} .
$$

In machine learning applications, it is more useful to represent DPP in terms of quality and diversity aspects. Let us consider a vector $\mathbf{e}_{i} \in \mathbb{R}^{K}$ to represent the i-th item of $\mathcal{K}$, the corresponding $\mathrm{E} \in \mathbb{R}^{K \times K}$ can be interpreted as a kernel ${ }_{210}$ matrix as $\mathrm{E}_{i, j}=\mathrm{k}\left(\mathbf{e}_{i}, \mathbf{e} j\right) \equiv \phi\left(\mathbf{e}_{i}\right) \phi^{\top}\left(\mathbf{e}_{j}\right)$, where $\phi$ is the corresponding feature map. Applying decomposition to the kernel matrix, $\mathrm{E}$ matrix is rewritten as follows:

$$
\mathrm{E}_{i, j}=\mathrm{k}\left(\mathbf{e}_{i}, \mathbf{e} j\right)=q\left(\mathbf{e}_{i}\right) S_{i, j} q\left(\mathbf{e}_{j}\right),
$$

where $q\left(\mathbf{e}_{i}\right)$ denotes the quality of $\mathbf{e}_{i}$ and $S_{i, j}=\mathrm{E}_{i, j} / \sqrt{\mathrm{E}_{i, i} L_{j, j}}$ denotes the similarity of $\mathbf{e}_{i}$ and $\mathbf{e}_{j}\left(\forall_{i, j} \in \mathcal{K}, i \neq j\right)$. We can use (14) to rewrite (12) after some manipulation as

$$
\mathcal{P}_{\mathrm{E}}(\mathbf{Y}=Y) \propto \operatorname{det}\left(\mathrm{E}_{Y}\right)=\operatorname{det}\left(S_{Y}\right) \prod_{i \in Y} q\left(\mathbf{e}_{i}\right)^{2},
$$


where $S_{Y}$ represents the diversity and $q(\mathbf{e})$ represents the quality of the items in $Y$.

Now, let us explain the geometry interpretation of $\mathcal{P}_{\mathrm{E}}(\mathbf{Y}=Y)$. Intuitively, geometric interpretation of the determinant is the squared volume of the parallelepiped spanned by the feature vectors $\phi\left(\mathbf{e}_{i}\right)$ which is an element in $\mathcal{K}$, where $\left\|\phi\left(\mathbf{e}_{i}\right)\right\|=q\left(\mathbf{e}_{i}\right)$ and $\angle\left\{\phi\left(\mathbf{e}_{i}\right), \phi\left(\mathbf{e}_{j}\right)\right\}=\arccos \left(S_{i, j}\right)$. Since DPP assigns the probability to a set $Y$ by evaluating the volume spanned by its related feature vectors, similar items are less probable than diverse items as less orthogonal collection of $\phi\left(\mathbf{e}_{i}\right)$ spans less volumes. Items with large $\left\|\phi\left(\mathbf{e}_{i}\right)\right\|$ are more probable because the volume spanned by its related feature vectors increases. Thus, the DPP decomposed as quality and diversity naturally balances these two objectives.

\section{THE PROPOSED DPPL FRAMEWORK}

\subsection{Conditional DPPs}

In many learning applications, input data is crucial. For example, extractive document summarization, samples of different sentences are required as input to perform a good summary of the entire article. Thus, the aim is to choose a subset of sentences from the article that can predict a good summary. This selected subset is used as a data input to obtain a conditional parameterized DPP. Let us consider $\left\{a_{k}^{*}\right\}$ as the external input, $\mathcal{K}\left(\left\{a_{k}^{*}\right\}\right)$ is the underlying set of all subsets implied by an input $\left\{a_{k}^{*}\right\}$. For each possible subset $Y \subseteq \mathcal{K}\left(\left\{a_{k}^{*}\right\}\right)$, there will be a probability assigned by a conditional DPP as

$$
\mathcal{P}_{\mathrm{E}}\left(\mathbf{Y}=Y \mid\left(\left\{a_{k}^{*}\right\}\right)\right) \propto \operatorname{det}\left(\mathrm{Ł}_{Y}\left(\left\{a_{k}^{*}\right\}\right)\right),
$$

where $\mathrm{E}_{Y}\left(\left\{a_{k}^{*}\right\}\right)$ is a positive semidifinite kernel matrix with dimension $\left|\mathcal{K}\left(\left\{a_{k}^{*}\right\}\right)\right| \times$ $\left|\mathcal{K}\left(\left\{a_{k}^{*}\right\}\right)\right|$. The normalization constant is $\operatorname{det}\left(\mathrm{E}\left(\left\{a_{k}^{*}\right\}\right)+I\right)$. By using the decomposition technique, we can rewrite the $\mathrm{E}$ matrix in terms of input $\left\{a_{k}^{*}\right\}$ as follows

$$
\mathrm{E}_{i, j}\left(\left\{a_{k}^{*}\right\}\right)=q\left(\mathbf{e}_{i} \mid\left\{a_{k}^{*}\right\}\right) S_{i, j}\left(\left\{a_{k}^{*}\right\}\right) q\left(\mathbf{e}_{j} \mid\left\{a_{k}^{*}\right\}\right),
$$

where the first part $q\left(\mathbf{e}_{i} /\left\{a_{k}^{*}\right\}\right)$ is the quality measure for the item $i$ and $S_{i, j}$ is the diversity measure of the items $i$ and $j(i \neq j)$. One of the commonly used expressions for the quality part parameterized by $\theta$ is the $\log$-linear model which was proposed in [10] as follows

$$
q\left(\mathbf{e}_{i} \mid\left\{a_{k}^{*}\right\}\right)=\exp \left(\theta^{\top} \mathbf{f}\left(\mathbf{e}_{i} \mid\left\{a_{k}^{*}\right\}\right)\right),
$$

where $\theta$ and $\mathbf{e}_{i}$ have the same dimension.

For our proposed model, the quality and diversity measures are set as $q\left(\mathbf{e}_{i} \mid\left\{a_{k}^{*}\right\}\right):=$ $\exp \left(\theta_{1} \mathbf{h}_{i} \tilde{\mathbf{w}}_{i}+\theta_{2} I_{1}+\theta_{3} I_{2}\right)$, where $I_{1}=\mathbf{h}_{i} \tilde{\mathbf{w}}_{j^{\prime}}$ with $j^{\prime}=\arg \max _{j=1, \ldots, L \neq i}\left\{\tilde{\mathbf{w}}_{j}\right\}$ and $I_{2}=\mathbf{h}_{i} \tilde{\mathbf{w}_{j^{\prime \prime}}}$ with $j^{\prime \prime}=\arg \max _{j=1, \ldots, L \neq i, j^{\prime}}\left\{\tilde{\mathbf{w}}_{j}\right\}$ are the strongest interfering 
power to the user $i$ and $S_{i, j}\left(\left\{a_{k}^{*}\right\}\right)=\left|\mathbf{h}_{i}^{H} \mathbf{h}_{j} /\left\|\mathbf{h}_{i}\left|\|\| \mathbf{h}_{j} \|\right|\right.\right.$, where $\tilde{\mathbf{w}}_{i}$ is the maximum ratio transmission beamforming for user $i, \mathbf{h}_{i}$ and $\mathbf{h}_{j}$ denote the channel vectors for user $i$ and $j$, respectively.

\subsection{Training $D P P$}

Let us consider a sequence of ordered pairs denoted as $\left(\left(\left\{a_{k}^{*}\right\}\right)^{(t)}, Y^{(t)}\right), t=$ $1,2, \ldots . T$ be the training samples, where $\left(\left\{a_{k}^{*}\right\}\right)^{(t)}$ is the input data and $Y^{(t)} \subseteq$ $\mathcal{K}\left(\left(\left\{a_{k}^{*}\right\}\right)^{(t)}\right)$ is the corresponding ground set for input $\left(\left\{a_{k}^{*}\right\}\right)^{(t)}$. These training samples are used to learn the parameters $\theta$. Hence, the learned parameters can provide an accurate prediction to any unseen input data. We assume the parameterized conditional kernel is $\mathrm{L}\left(\left\{a_{k}^{*}\right\} ; \theta\right)$. Following $[12$, for any given input $\left\{a_{k}^{*}\right\}$, the conditional probability of output $Y$ can be obtained as

$$
\mathcal{P}_{\theta}\left(Y \mid\left\{a_{k}^{*}\right\}\right)=\frac{\operatorname{det}\left(\mathrm{E}_{Y}\left(\left\{a_{k}^{*}\right\} ; \theta\right)\right)}{\operatorname{det}\left(\mathrm{E}\left(\left\{a_{k}^{*}\right\} ; \theta\right)+I\right)} .
$$

Now, we formulate the learning problem as the maximization of the loglikelihood of the trained conditional DPPs as follows

$$
\begin{aligned}
& \left(\theta^{*}\right)=\max _{(\theta)} \sum_{t=1}^{T} \log \mathcal{P}_{\theta}\left(Y^{(t)} \mid\left(\left\{a_{k}^{*}\right\}\right)^{(t)}\right) \\
& =\sum_{t=1}^{T}\left[\log \operatorname{det}\left(\mathrm{E}_{Y^{(t)}}\left(\left(\left\{a_{k}^{*}\right\}\right)^{(t)} ; \theta\right)\right)\right. \\
& \left.-\log \operatorname{det}\left(\mathrm{E}\left(\left(\left\{a_{k}^{*}\right\}\right)^{(t)} ; \theta\right)+I\right)\right] .
\end{aligned}
$$

The reason for choosing the log-linear model for quality measure is due to the fact that under this model, the optimization problem in $(20)$ is a convex problem because $\sum_{t=1}^{T} \log \mathcal{P}_{\theta}\left(Y^{t} \mid\left(\left\{a_{k}^{*}\right\}\right)^{t}\right)$ becomes a concave function of $\theta$.

\subsection{Inference}

The parameter $\theta$ which is obtained from learning conditional DPP is used to predict $\left\{\hat{a_{k}^{*}}\right\}$ for any given $\left\{a_{k}^{*}\right\}$. This phase is also known as the testing phase. To predict $\left\{\hat{a_{k}^{*}}\right\}$, sampling from DPP is one of the methods which are used. The main idea is to choose a random sample from the DPP, i,e,. $\mathbf{Y} \sim \mathcal{P}_{\theta^{*}}\left(. \mid\left\{a_{k}^{*}\right\}\right)$ and set $\left\{\hat{a_{k}^{*}}\right\}=\mathbf{Y}$. We aim to draw a random samples for a general DPP and then extend it to a conditional DPP. We first start drawing a random samples from a special case of DPP which is known as the elementary DPP and then use this scheme to draw a samples from the general DPP. We call a DPP on

the elementary DPP if all the eigenvalues of its marginal kernel matrix are either 0 or 1 . We denote the elementary DPP as $\mathcal{P}^{V}$ where $V$ is the set of orthonormal vectors. Thus, the marginal kernel matrix of the elementary DPP is $\kappa^{V}=\sum_{\mathbf{v} \in V} \mathbf{v v}^{\top}$. The sampling procedure was proposed in [20]. There are two key steps: 
- Sample $\left\{\hat{a_{k}^{*}}\right\}$ from $\mathcal{P}^{V}$.

In the first step, we randomly select a subset of eigenvectors with the probability $\frac{\lambda_{k}}{1+\lambda_{k}}$ and in the second step, we sample a subset $\left\{\hat{a_{k}^{*}}\right\}$ from the corresponding elementary DPP to the eigenvectors which are selected in the first step (see 10, Lemma 2.6 and Lemma 2.7 for proof). Algorithm 1 describes the sampling procedure.

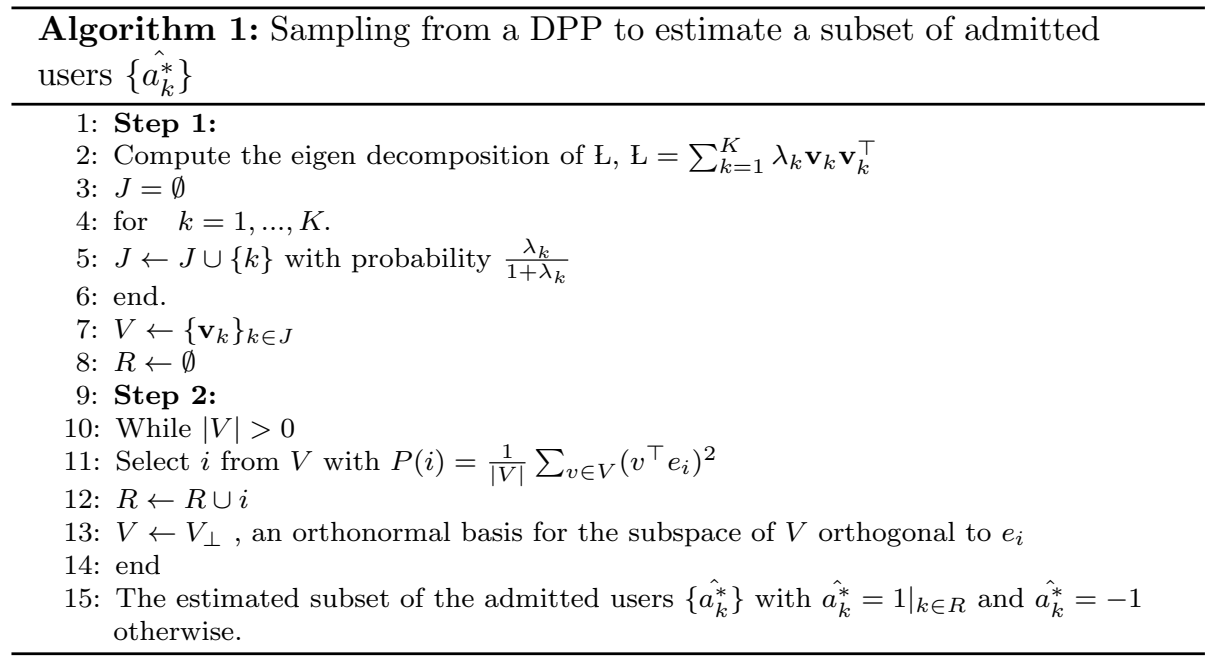

The output $\left\{\hat{a_{k}^{*}}\right\}$ in Algorithm 1 denotes the estimated subset of the admitted users. To obtain the beamforming and base station-user allocation matrix, we use the optimization problem in (9p). Due to the fact that the estimated subset of the admitted users which is obtained from Algorithm 1 will not always satisfy the constraints in (9), we propose Algorithm 2 to ensure the problem feasibility and to obtain the beamforming and the base station-user allocation for the problem (9). For any obtained an estimated subset of the admitted users, we generate the base station-user allocation matrix such that when $\hat{a_{k}^{*}}=1, \hat{\mathbf{b}_{k}^{*}}=\mathbf{1}$, and $\hat{a_{k}^{*}}=-1, \hat{\mathbf{b}_{k}^{*}}=\mathbf{0}$ and named it Solution. We solve the problem in (9) with the user admission and the base station-user allocation (Solution). If the solution is feasible, we calculate the base station normalized power for each admitted user. If the normalized power is less than the threshold value, then, we set the value of the base station-user allocation for the particular user to 0 , i.e., $295 \hat{b}_{l, k}^{*}=0$. We solve the problem in (9) with the updated values of $\hat{b}_{l, k}^{*}$ to obtain the beamforming. If the solution is infeasible, we solve the problem in 9 with relaxed values of integer constraints. We calculate the SINR for each user and sort SINR values in the decreasing order. We set the values of the user admission and base station-user allocation for the user index with minimum SINR to $300-1$ and $\mathbf{0}$, respectively, i,e,. $\hat{a}_{k}^{*}=-1, \hat{\mathbf{b}_{k}^{*}}=\mathbf{0}$ and update the Solution. We solve the problem in (9) with an updated Solution and repeat the previous procedure 
until the algorithm is terminated.

The convergence speed for the proposed algorithms can be determined by the number of subproblems needs to be solved for each algorithm. Since each of these subproblems is a SOCP with $K L$ linear constraints, it requires $O[\sqrt{K L} \log (1 / \epsilon)]$ iterations with $\epsilon$ solution accuracy for the convergence of the algorithm using the interior point method. In the worst case, the optimal BnB solution requires $\sum_{k=1}^{K}\left(2^{L}\right)^{k}$ subproblems to be solved whereas the number of subproblems re310 quired for the suboptimal BnB solution is $K\left(2^{L}\right)$. In contrast, our proposed DPPL based solution only requires $K$ subproblems to be solved, consequently, the complexity is reduced greatly.

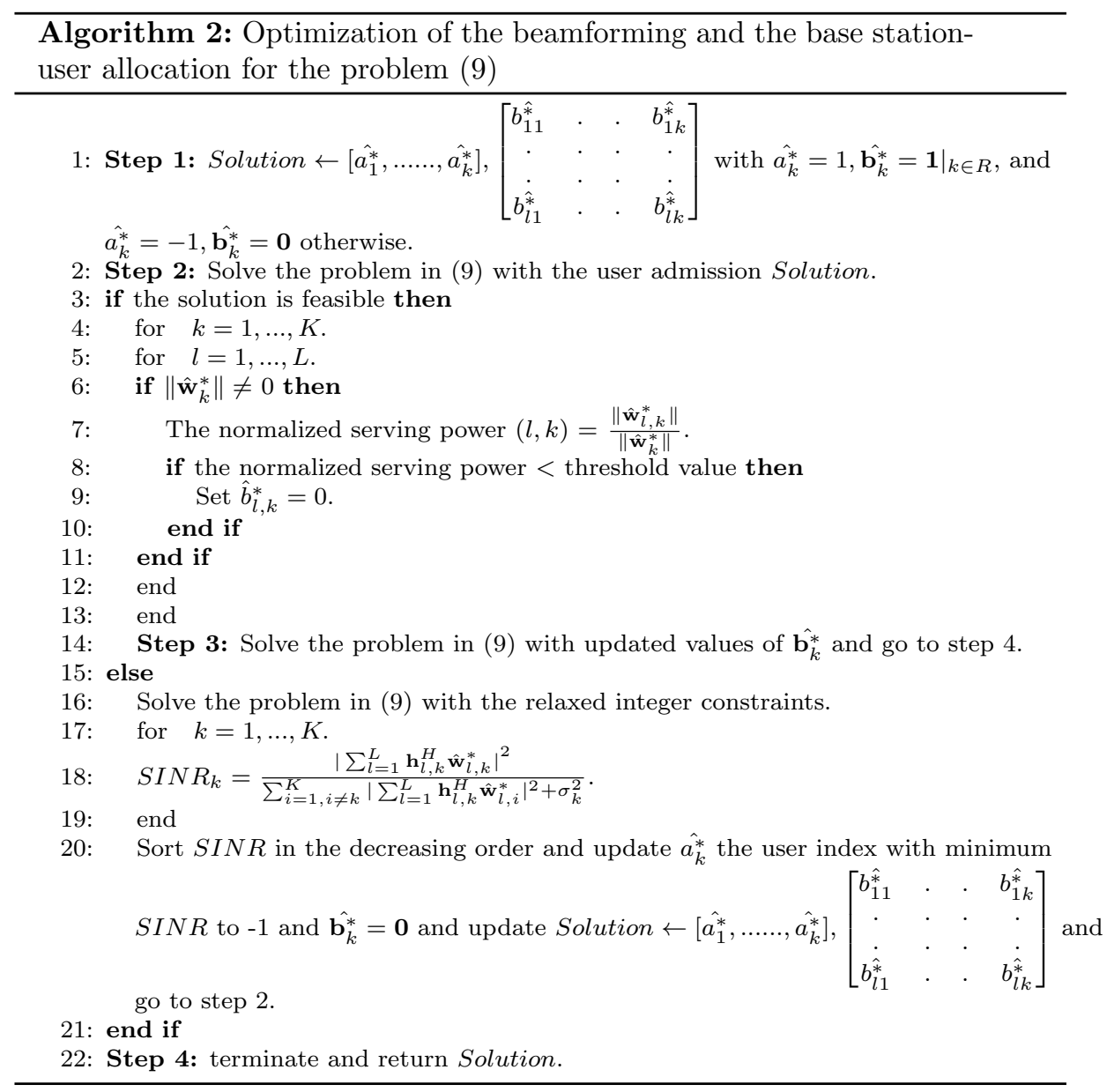




\section{SIMULATION RESULTS}

315 $L=2$ ), each equipped with $N=3$ antennas and $K=6$ users. We consider an identical target SINR requirement for all the users. The channels between the RRHs and the users are generated using a Rayleigh fading model. Each entry of the channel vector is identically and independently distributed symmaximum transmit power at the RRH $\left(P_{l}^{M}\right)$ is 5 Watt. All RRHs are assumed to have equal cache size. The available channel bandwidth is $5 \mathrm{MHz}$. We set the parameter $\alpha$ equal to 0.01 and the constant $\eta$ in the problem (6) equal to 1. The maximum fronthaul link capacity of $\mathrm{RRH} l$ link $\left(R_{l}^{M}\right)$ is $80 \mathrm{Mbps}$. Each 325 user submits contents request independently. Different users cannot request the same content simultaneously. The cache placement matrix is known at RRHs. We generate 200 network realizations for training purpose, i.e., to train the conditional DPPs using the optimal subsets of admitted users and 100 network realizations for testing. Each simulation result is based on the average of 100

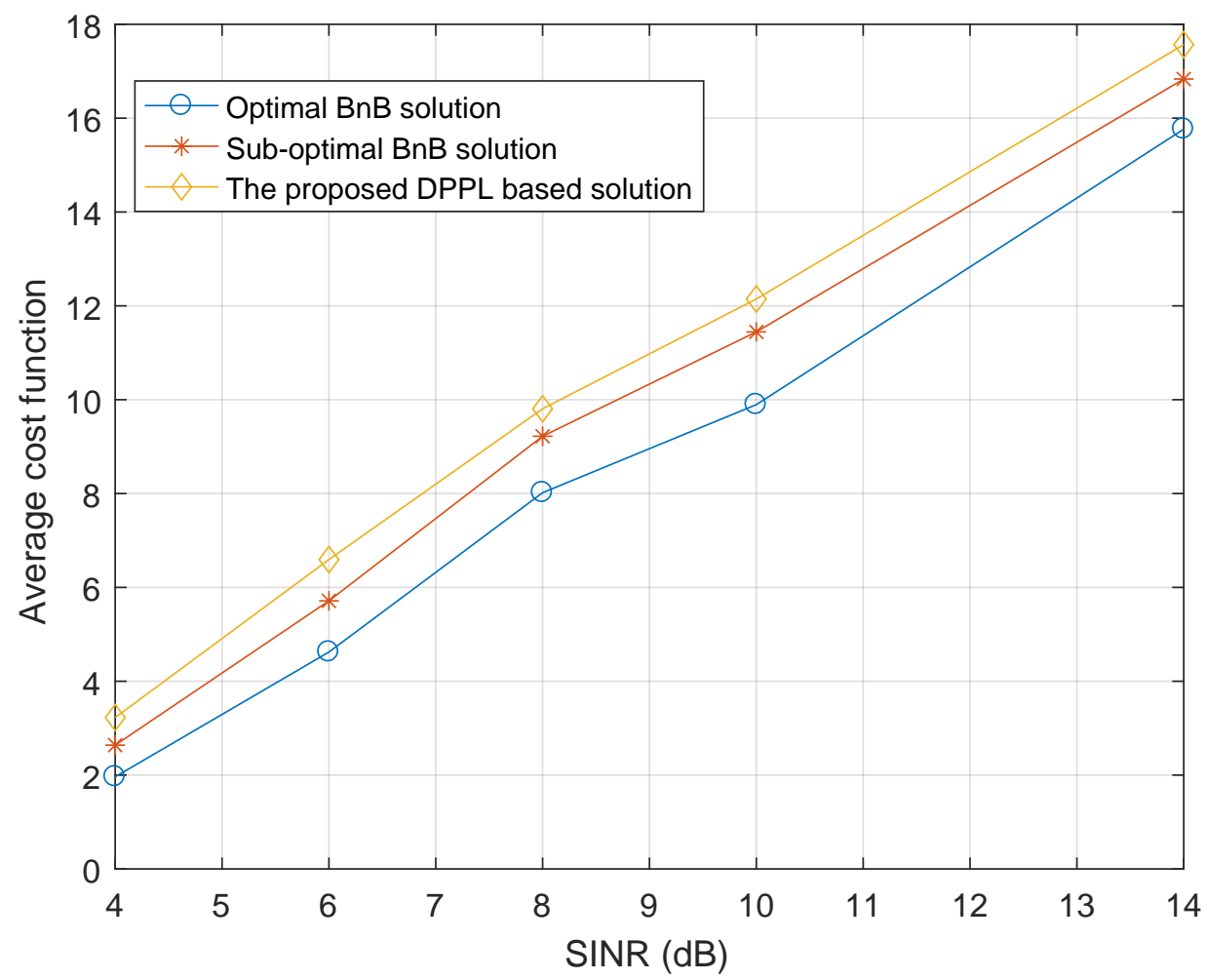

Figure 2: Average cost function versus target SINR, $L=2, N=3, K=6$. 


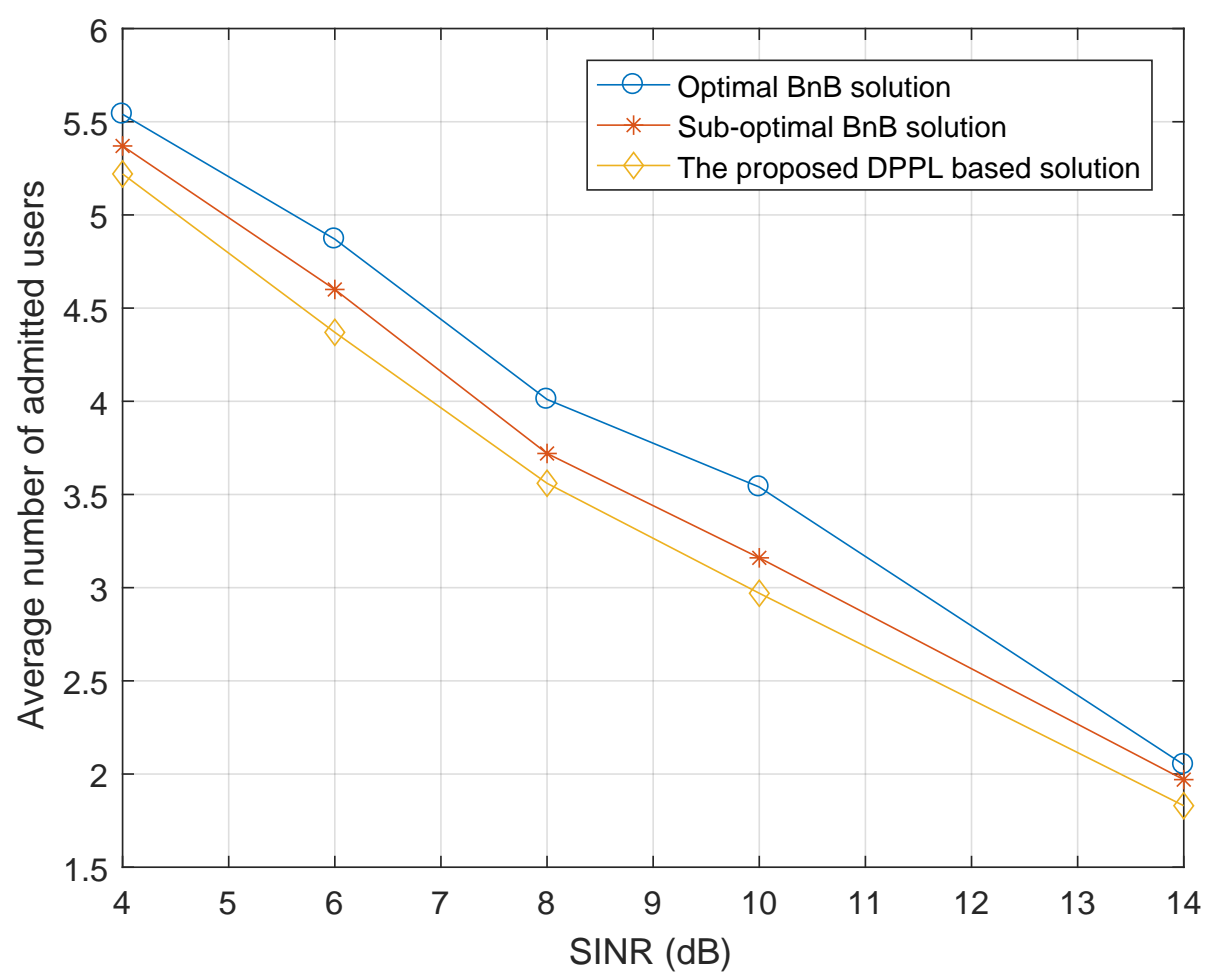

Figure 3: Average number of admitted users versus SINR, $L=2, N=3, K=6$. 


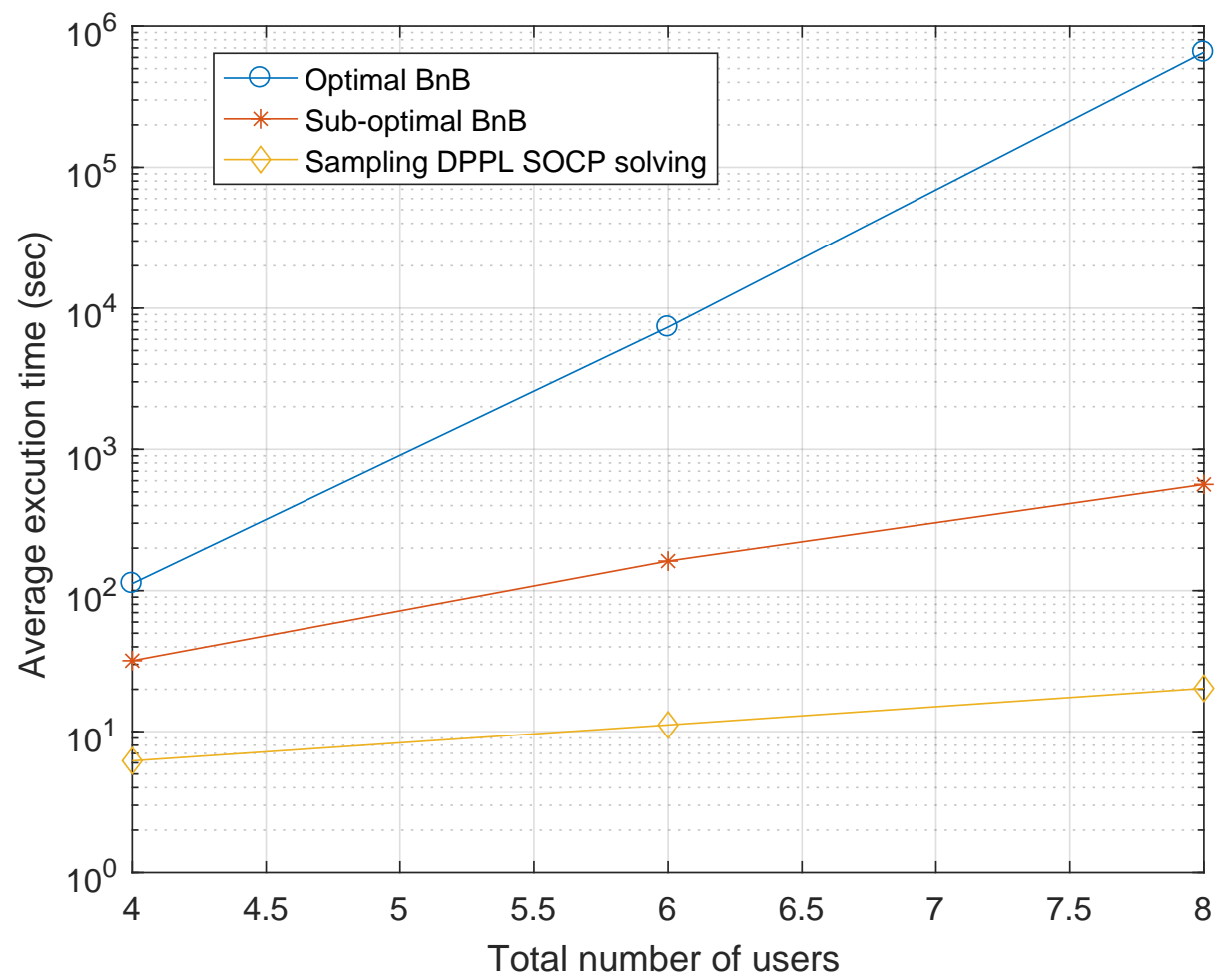

Figure 4: Average execution time versus total number of users, $L=2, N=3$, SINR $=10 \mathrm{~dB}$.

We demonstrate the performance of our proposed DPPL framework using the same system model configuration for both training and testing phases. We compare the performance of our proposed DPPL framework with the state of the art $\mathrm{BnB}$ algorithms. We choose the optimal and sub-optimal BnB Algorithms as benchmarks [18. Figure 2 and Figure 3 show the performance in terms of the average cost function and the average number of admitted users versus the target SINR, respectively. Although the optimal BnB algorithm is able to admit more users and require lower cost, our proposed DPPL framework algorithm has the advantage of requiring much lower complexity while achieving a comparable performance. We assess the execution running time for each algorithm at a target SINR of $10 \mathrm{~dB}$ as shown in Figure 4 Although the optimal algorithm provides an attractive low cost function solution, it requires a long time for processing compared to the proposed DPPL framework. Hence, our proposed DPPL framework provides a promising trade-off between the optimal solution and computational complexity. The results show some degree of generalization capability of our proposed DPPL framework.

To investigate the generalization capability of our proposed DPPL frame- 

$\mathrm{BnB}$ approach. We observe that the proposed DPPL framework still show a good generalization ability even if trained on different network configuration settings.

Table 1: Average cost function

\begin{tabular}{|c|c|c|}
\hline Setting & DPPL & Sub-optimal BnB \\
\hline$L=3, K=6$, SINR $=4 \mathrm{~dB}$ & 0.6344 & 0.5597 \\
\hline$L=4, K=8$, SINR $=4 \mathrm{~dB}$ & 0.8124 & 0.1861 \\
\hline$L=5, K=10$, SINR $=4 \mathrm{~dB}$ & 0.5690 & 0.0972 \\
\hline
\end{tabular}

Table 2: Average number of admitted users

\begin{tabular}{|c|c|c|}
\hline Setting & DPPL & Sub-optimal BnB \\
\hline$L=3, K=6$, SINR $=4 \mathrm{~dB}$ & 5.86 & 5.88 \\
\hline$L=4, K=8$, SINR $=4 \mathrm{~dB}$ & 7.82 & 7.98 \\
\hline$L=5, K=10$, SINR $=4 \mathrm{~dB}$ & 9.88 & 10.00 \\
\hline
\end{tabular}

\section{CONCLUSION}

In this paper, we have developed a Determinantal point process learning framework to solve a subset selection problem in cache-enabled Cloud-RAN with limited fronthaul capacity. The JBAC problem was formulated as minimizing the total network cost including the power cost and the fronthaul cost while admitting as many users as possible under certain constraints. The formulated problem was transformed after relaxing the integer variables into the MI-SOCP form. The estimated subset of admitted users was obtained by training the DPPs using the BnB optimal solution. We proposed Algorithm 2 to optimize the beamforming and the base station-user allocation. Simulation results have shown that the proposed DPPL framework achieves a comparable performance 370 with significant complexity reduction compared to the conventional $\mathrm{BnB}$ approaches.

\section{References}

[1] M. A. Habibi, M. Nasimi, B. Han, H. D. Schotten, A comprehensive survey of ran architectures toward $5 \mathrm{G}$ mobile communication system, IEEE Access 7 (2019) 70371-70421. doi:10.1109/ACCESS. 2019.2919657.

[2] L. Li, G. Zhao, R. S. Blum, A survey of caching techniques in cellular networks: Research issues and challenges in content placement and delivery 
strategies, IEEE Communications Surveys Tutorials 20 (3) (2018) 17101732. doi:10.1109/COMST.2018.2820021.

380

[3] B. Dai, W. Yu, Energy efficiency of downlink transmission strategies for cloud radio access networks, IEEE Journal on Selected Areas in Communications 34 (4) (2016) 1037-1050. doi:10.1109/JSAC.2016.2544459.

[4] K. Wang, K. Yang, C. S. Magurawalage, Joint energy minimization and resource allocation in C-RAN with mobile cloud, IEEE Transactions on Cloud Computing 6 (3) (2018) 760-770. doi:10.1109/TCC.2016.2522439

[5] S. Kuang, N. Liu, Energy minimization via BS selection and beamforming for Cloud-RAN under finite fronthaul capacity constraints, in: 2016 IEEE 83rd Vehicular Technology Conference (VTC Spring), Nanjing, China, 2016, pp. 1-6. doi:10.1109/VTCSpring.2016.7504227.

[6] D. Pompili, A. Hajisami, T. X. Tran, Elastic resource utilization framework for high capacity and energy efficiency in Cloud RAN, IEEE Communications Magazine 54 (1) (2016) 26-32. doi:10.1109/MCOM.2016.7378422

[7] X. Wang, S. Thota, M. Tornatore, H. S. Chung, H. H. Lee, S. Park, B. Mukherjee, Energy-efficient virtual base station formation in opticalaccess-enabled Cloud-RAN, IEEE Journal on Selected Areas in Communications 34 (5) (2016) 1130-1139. doi:10.1109/JSAC.2016.2520247.

[8] H. Sun, X. Chen, Q. Shi, M. Hong, X. Fu, N. D. Sidiropoulos, Learning to optimize: Training deep neural networks for interference management, IEEE Transactions on Signal Processing 66 (20) (2018) 5438-5453. doi: 10.1109/TSP.2018.2866382

[9] O. Simeone, A very brief introduction to machine learning with applications to communication systems, IEEE Transactions on Cognitive Communications and Networking 4 (4) (2018) 648-664. doi:10.1109/TCCN. 2018. 2881442

[10] A. Kulesza, B. Taskar, et al., Determinantal point processes for machine learning, Foundations and Trends $\mathbb{R}$ in Machine Learning 5 (2-3) (2012) 123-286. doi:10.1561/2200000044.

[11] Y. Li, F. Baccelli, H. S. Dhillon, J. G. Andrews, Statistical modeling and probabilistic analysis of cellular networks with determinantal point processes, IEEE Transactions on Communications 63 (9) (2015) 3405-3422. doi:10.1109/TCOMM.2015.2456016

[12] N. Miyoshi, T. Shirai, A cellular network model with Ginibre configured base stations, Advances in Applied Probability 46 (3) (2014) 832-845. doi: 10.1239/aap/1409319562. 
[13] B. Blaszczyszyn, H. P. Keeler, Determinantal thinning of point processes with network learning applications, in: 2019 IEEE Wireless Communications and Networking Conference (WCNC), Marrakesh, Morocco, 2019, pp. 1-8. doi:10.1109/WCNC.2019.8885526.

[14] C. Saha, H. S. Dhillon, Machine learning meets stochastic geometry: Determinantal subset selection for wireless networks, in: 2019 IEEE Global Communications Conference (GLOBECOM), Waikoloa, HI, USA, 2019, pp. 1-6. doi:10.1109/GLOBECOM38437.2019.9013889.

[15] M. Tao, E. Chen, H. Zhou, W. Yu, Content-centric sparse multicast beamforming for cache-enabled Cloud RAN, IEEE Transactions on Wireless Communications 15 (9) (2016) 6118-6131. doi:10.1109/TWC.2016. 2578922

[16] E. Matskani, N. D. Sidiropoulos, Z. Q. Luo, L. Tassiulas, Convex approximation techniques for joint multiuser downlink beamforming and admission control, IEEE Transactions on Wireless Communications 7 (7) (2008) 2682-2693. doi:10.1109/TWC.2008.070104

[17] K. Cumanan, R. Krishna, L. Musavian, S. Lambotharan, Joint beamforming and user maximization techniques for cognitive radio networks based on branch and bound method, IEEE Transactions on Wireless Communications 9 (10) (2010) 3082-3092. doi:10.1109/TWC.2010.072610.090898

[18] A. Bsebsu, G. Zheng, S. Lambotharan, K. Cumanan, B. AsSadhan, Joint beamforming and admission control for cache-enabled cloud-ran with limited fronthaul capacity, IET Signal Processing 14 (5) (2020) 278-287. doi:10.1049/iet-spr.2019.0247.

[19] A. Borodin, E. M. Rains, Eynard-mehta theorem, schur process, and their pfaffian analogs, Journal of statistical physics 121 (3-4) (2005) 291-317. doi:10.1007/s10955-005-7583-z.

[20] J. B. Hough, M. Krishnapur, Y. Peres, B. Virág, et al., Determinantal processes and independence, Probability surveys 3 (2006) 206-229. doi: 10.1214/154957806000000078. 